\title{
DESIGN OF DIFFERENT TOPOLOGIES IN SDN CONTROLLER
}

\author{
Noora V T \\ Research Scholar, Department of Computer Science and Engineering, \\ Noorul Islam Centre for Higher Education, Kumaracoil, \\ Nagercoil, Tamilnadu,Pin-629180, India \\ nooravt@gmail.com \\ https://orcid.org/0000-0003-0604-8788 \\ Dr. S Vinila Jinny \\ Research Scholar, Department of Computer Science and Engineering, \\ Noorul Islam Centre for Higher Education, Kumaracoil, \\ Nagercoil, Tamilnadu,Pin-629180, India \\ vinijini@gmail.com \\ https://orcid.org /0000-0003-2756-6736
}

\begin{abstract}
G is the most recent advancement of cellular communications. Due to its considerable advantages in high data rate, decreased latency and huge gadget connectivity, it has an indispensable role in the present media communications systems. Difficulties on account of different assorted ingredients, named heterogeneity. To overcome this restriction, software-defined networking(SDN) is the idea. The knowledge centralization utilized in SDN has disadvantages in security. To overcome, we propose a vigorous security design for SDN-based 5G Networks using controller(SDNC).By utilizing this incorporated controller between the data plane and the control plane, time can be decreased. At last by utilizing open flow controller, various topologies, for example, single, linear, tree, reversed and minimal are thought about and distinguished that direct topology has higher data transfer capacity and demonstrated better inactivity of 0.07 seconds. This work measure the quality factors in the versatile correspondence system, for example, idleness, data transfer capacity and throughput.
\end{abstract}

Keywords: Software defined networking controller (SDNC); 5G; Openflow;Mininet topologies.

\section{Introduction}

5G systems, as another remote correspondence innovation, experience a quick improvement as of late. As appeared in Fig. 1, this system has been broadly utilized in each edge of our day by day life. Contrasted and the obsolete business 4G (LTE/WiMax) framework, this innovation has focal points in high information rate, diminished inertness, and huge gadget network, making it a basic foundation module for remote correspondence sooner rather than later. Propelled by its critical points of interest, numerous specialists have structured their own conventions to make it fit the prerequisite of the genuine applications. Right now, characterized organizing (SDN) is one of the key plan ideas(ETSI MCC Department,2014).

SDN is a way to deal with improve organize execution and observing by encouraging system the board and empowering automatically proficient system setup. By isolating information and control planes, SDN empowers a wide scope of new imaginative applications from traffic building to server farm virtualization, fine-grained get to control, etc(ETSI MCC Department,2013) . It has demonstrated preferred position in numerous business systems and in this way is additionally a decent decision in the field of $5 \mathrm{G}$ systems. Regardless of these points of interest, shaping such a SDN-based 5G organize isn't free, and there remains a ton of difficulties with regards to security. This is on the grounds that the insight centralization of SDN is defenseless for different assaults. A few research deals with SDN have just explored security applications based upon the SDN controller, in view of various points. Dispersed Denial of Service (DDoS) recognition and alleviation, just as botnet and worm spread, are some solid use-instances of such applications: essentially, the thought comprises of intermittently gathering system measurements from the sending plane of the system in an institutionalized way (e.g., utilizing OpenFlow), and afterward apply arrangement calculations on those insights so as to distinguish any system inconsistencies(J. Mueller, Y. Chen, B. Reichel, V. Vlad and T. Magedanz,2014). On the off chance that a peculiarity is distinguished, the application trains the controller how to reconstruct the information plane so as to relieve it. The SDN controller is also used by another type of security program by modifying some moving objective safety calculations (MTD). In order to make a given framework or system more challenging than the one expected, the use of MTD calculations frequently increases or changes the key features of that framework or system. Upgrading MTD calculations in customary systems is certainly not a mismatch, since it is difficult to create a focal position fit which is suitable for evaluating the key features are concealed or modified for each part of the structure to be 
protected. In a SDN arrange, such assignments become increasingly clear gratitude to the centrality of the controller. One application can, for instance, occasionally dole out virtual IPs to inside the system, and the mapping virtual IP/genuine IP is then performed by the controller.

Another application can mimic some phony opened/shut/sifted ports on irregular has in the system so as to include critical clamor during the observation stage (e.g., filtering) performed by an aggressor(METIS,2013). Extra worth with respect to security in SDN empowered systems can likewise be picked up utilizing Flow Visor and Flow Checker, separately. The previous attempts to utilize a solitary equipment sending plane sharing different isolated consistent systems. Following this methodology, a similar equipment assets can be utilized for creation and advancement purposes just as isolating checking, arrangement and web traffic, where every situation can have its own intelligent topology which is called cut. Related to this methodology, FlowChecker understands the approval of new OpenFlow decides that are sent by clients utilizing their own cut. SDN controller applications are for the most part sent in huge scope situations, which requires complete checks of conceivable programming blunders. A framework to do this called NICE was portrayed in 2012. Presenting an all-encompassing security engineering requires a far reaching and extended way to deal with SDN. With the fast advancement of SDN, an ever increasing number of studies are completed to improve SDN organize execution and security(European Commission,2013).

The incorporated SDN engineering makes organize the executives significantly more simpler and flexible than previously, however it presents new security challenges as well. Along these lines, numerous securitysituated arrangements were proposed. OpenFlow system or Software Defined Network speak to another idea of systems administration that permits decoupling the control usefulness of sending gadgets, for example, switches into a focal rationale gadget called OpenFlow controller. SDN separates the usefulness of system gadgets into control plane and information plane (P. K. Agyapong, M. Iwamura, D. Staehle, W. Kiess and A. Benjebbour,2014). The control plane is installed inside the OpenFlow controller and it fills in as the focal unit controlling information stream sections in the change stream table to take into consideration fruitful conveyance of bundle. This is anyway not a similar procedure acted in customary systems. Each switch in a customary system performs control capacities and it is accepted that this capacities include overhead which restrains the exhibition of the system.

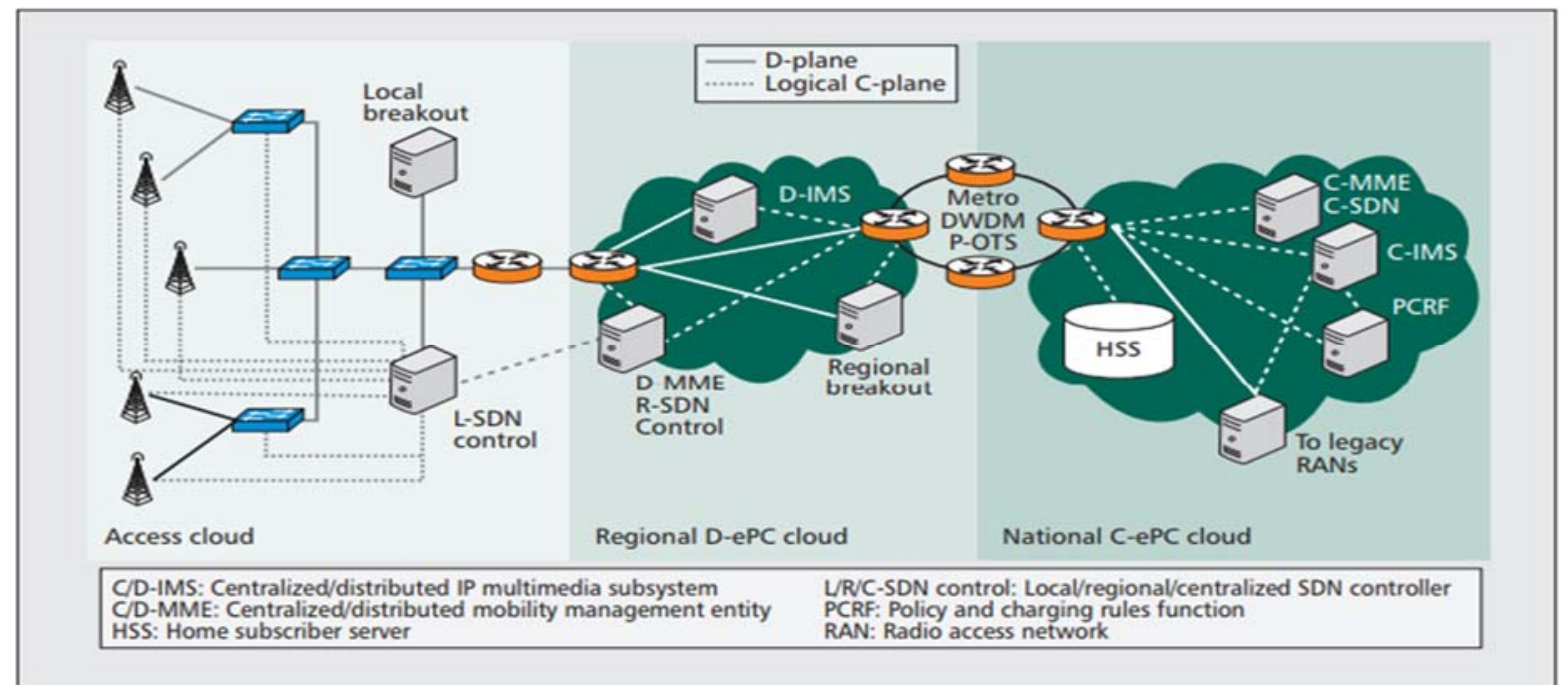

Fig 1.5G Architecture

Right now, focus at taking care of the security issues in SDN-based 5G systems Pablo Ameigeiras; et al(2015). As referenced, the SDN 5G arrange has two planes: the control plane and the information plane. We include a security module between the two planes. This security will synchronize privileged insights between the physical layer gadgets and the SDN controller(Ernardos, C.J. De La Oliva, A Serrano, P. anchs, A Contreras, L.M. Hao Jin niga, J.C,2014, V. Yazıc1, U. C. Kozat and M. O. Sunay,2014). The SDNC can be executed in openflow controller that is equipped with restricted time. SDNC, a strategy which is used to be controlled midway by both data as well as control plane. With help of sdn in mininet, copying can be carried out. Compared with different emulators, Mininet has built a good networking emulator with its switches, hosts and controller. Mininet bolster virtualization and it likewise bolster genuine system with genuine switch portion and application program.minine is absolutely founded on direction Line interpretation(CL1) mininet support openflow arrange for openflow processing( D. Soldani, A. Manzalini,2014). Versatile correspondence has become a significant piece of our life. So as to improve the quality variables of cell phones another innovation named Software Defined Networking Controller(SDNC) can be utilized by and by we are utilizing $4 \mathrm{G}$ innovation yet it is having less quality components for bandwidth, latency and throughput. 


\section{SDN Structure}

In the last couple of years there has been a significant increase over web usage. It has been an adjustment in the utilization of systems administration innovation that has been utilized a lot of now a days. The exercise of the adjustments in systems administration for the innovations has been mostly used(3GPP TS 23.401). In order to meet present requirements SDN has strengthened with standard program administration. SDN may also be better updated in many ways. Now using the distributed computer technologies, although fairly expected to extend and mechanize simple designs(R. Guerzoni R. Trivisonno, I. Vaishnavi, Z. Despotovic, A. Hecker, S. Beker, D. Soldani, 2014 ) Thanks to the methodology of the Open Network (ONF), SDN is indeed a reallocation of the both control as well as sending planes. SDN can be revised as the best method for operating organizations using a significant level of language to make the programming as adaptable as possible in order to increase the demand for distributed computer innovation(Rowshanrad, S., Abdi, V., \& Keshtgari, M ,2016). The SDN framework includes a controller and information plane to shape traditional device administration, openflow switching and SDN controller.

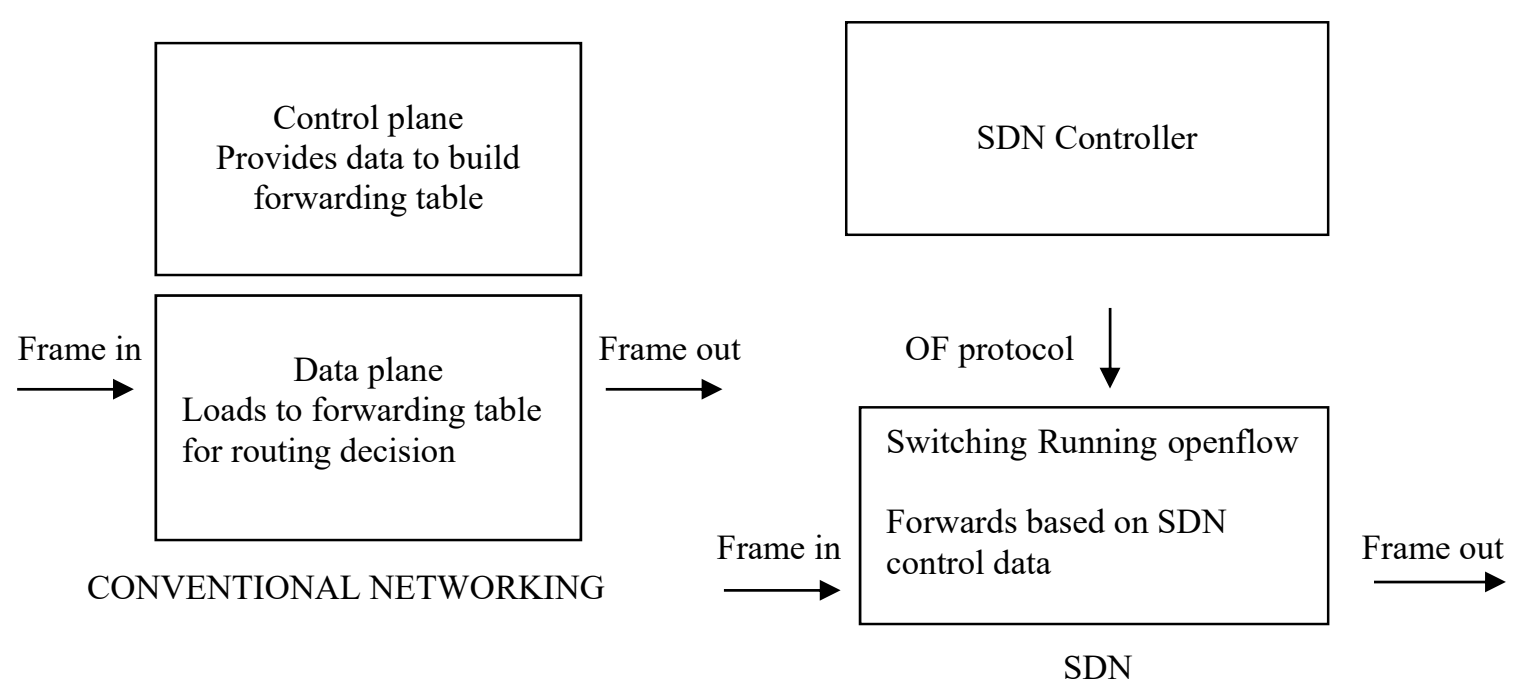

Fig. 2. SDN Structure

\section{SDN Model}

The execution model of SDN as appeared in Fig. 2 comprises of equipment, OF switch, hosts and controller for SDN. The SDN arrange associated with OF switch through openflow controller and controller again interfaces with equipment i.e., it comprises of meter table stream table and a product part it comprises of secure channel this equipment part associated with the hosts and the equipment part again associated into OF switch (Huiqiang Yuwen, Liancheng Zhang, Zhenxing Wang and Yazhou Kong,2016). The exemplary SDN design incorporates three virtual layers, control plane, information plane, and application plane. The application plane conjures sofware-based rationale in the control plane by means of REST-APIs which is short form of RepresentationalState Transfer Application Programming Interface which is additionally called Northbound Interface. The sent rationale choices are executed by the information plane through Southbound Interfaces, e.g., OpenFlow, a generally embraced usage engineering of SDN. The system control traffic is moved from the framework (information plane) to the controller (control plane). With the assistance of SDN applications, organize administrators accomplish recognized properties of system control, robotization and asset enhancement. Focal controller which is the place was all the information resides on control planes (Z. Zhao, F. Liu, D. Gong, L. Chen, F. Xiang and Y. Li,2017). Subsequently, OpenFlow organize design was adverted in midway activity engineering. Program which is set inside this is known as NOS which is a medium between information and application planes. This has about three layers. The information sending one is present in limited and only they have at least one open flow switches. Second layer will be control plan which consist of Open flow control with specific NOS(S. ShiraliShahreza and Y. Ganjali,2018). The third will be application plane which has atleast one open flow which has specific feature of executives or information streams dominant assignment. 


\section{Mininet}

Mininet is a device trigger which is used to create realistic hosts, electrical switch, controllers and link in systems. Mininet can run on regular Linux schedules, and it also uses switches that improve openflow for tailored, customized, deeply adaptable management and organizational programming(S. Dotcenko, A. Vladyko and I. Letenko,2014). The mininet VM is used to speed up the mininet configuration and is easy to use on non-linux processes. Mininet allows you to create different routing protocols with OpenFlow switches and has (end gadgets).Topologies will vary significantly from solo changes to slowly intertwined topology with different buttons and connections. You also can make topologies of your own. You can send traffic from among hosts and then display strands on the switches, and the digitally converted switches and acts like real gadgets(R. Mohammadi, R. Javidan and M. Conti,2017).Frameworks such as iperf can also be used to calculate system execution as it can be used for many applications. Unit topologies are performed using the "sudo $\mathrm{mn}$ " approach. For example, "- topo = linear,4" various alternatives can be utilized. It makes for a device consisting of 4 consecutive switches with each switch being associated with a solitary host which is associated with it.The VM works here on Windows Linux, through VM product, virtual box. Right now.

- VM establishment

- Virtual box

- Server Putty and Xming

It worked here having windows 7 with a 8 GB RAM VM Virtualbox. The mininet can be managed by running Prophet VM virtualbox. It renders with new extend and records, then the virtual box machine starts. Mininet provides an easy way to get correct system behavior ( and to the extent supported by the hardware performance) and to experiment with topologies. Minimet networks run real code including standard Unix/Linux network applications as well as the real Linux kernel and network stack ( including any kernel extensions which you may have available, as long as they are compatible with network namespaces) (Rietz, A. Brinner, and R. Cwalinsky,2015). Because of this, the code you develop and test on Mininet, for an Openflow controller, modified switch, or host, can move to a real system with minimal changes, for real-world testing, performance evaluation and deployment. Importantly this means that a design that works in Mininet can usually move directly to hardware switches for line-rate packet forwarding.

\section{Open Flow Network Topology}

System network topology are the course action of hubs in a system in few particular way. Hubs may incorporate last clients, arrange center gadgets such as centers, switches, and so forth. It might likewise incorporate printing machine, digital scanner, telefax device, and so forth (J. H. Cox, R. J. Clark, and H. L. Owen,2016). The link of every gadgets used here in some physical style shapes in cooperates system topology.

Not at all like conventional systems administration engineering, in OpenFlow-empowered systems. Right now, OpenFlow-empowered system design for fundamental OpenFlow arranges topologies are actualized utilizing Mininet: A quick prototyping for programming characterized organize(J. Wang and Y. Chen,2017). The regular topology is an insignificant topology, which is used to be predefined with one OpenFlow bit change linked with two hosts. OpenFlow reference controller, although the number of switches can be changed for different topologies by utilizing the direction line interface (CLI). The three fundamental OpenFlow organize topologies are discussed here. The presentation investigation of a system comparative with transmission capacity usage, deferral of the system, generally arranges throughput and burden on the system is finished. 


\section{Implementation Diagram of Proposed Network}

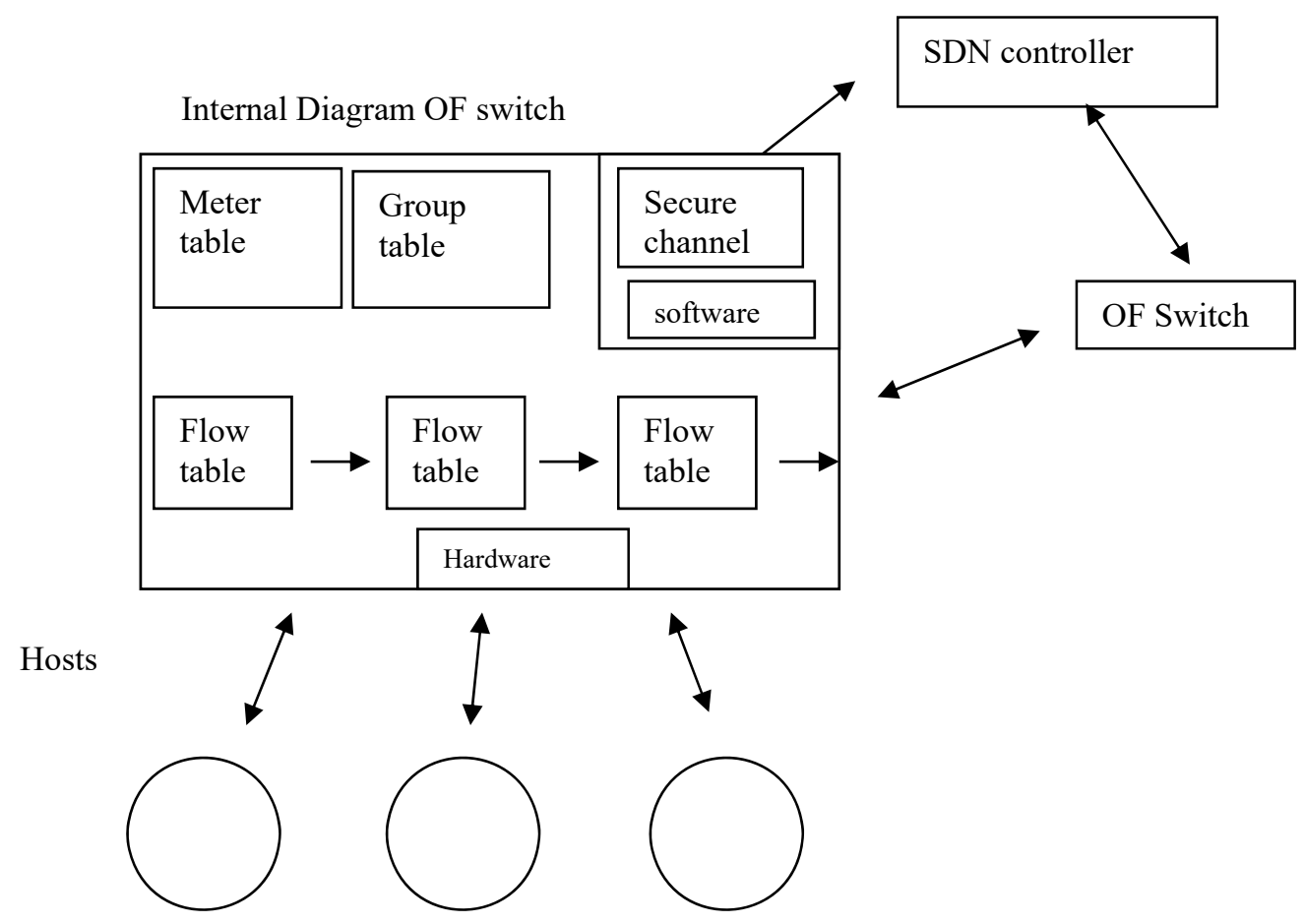

Fig. 3. Implementation model

In the SDN controller, it is worked by utilizing openflow organize. Switch contains diverse host associated with the stream table, meter table, group table and secure channel. It is associated with Ofswitch It again associated with openflow organize and to SDN controller. The SDN controller has been executed in Mininet. Mininet has been impemented in Oracle Virtual Box Implement in openflow. OpenFlow Controller can deal with a lot of gear while keeping up an elevated level of administration. The effect of the inactivity of the change to-controller connect on the exhibitions of the entire system is assessed. An OpenFlow Controller is equipped for preparing near a million streams for every second (A.S. D. Alfoudi, S. H. S. Newaz, R. Ramlie, G. M. Lee and T. Baker,2019). It is along these lines not a bottleneck for the exhibitions of the entire system. We indicated the data transfer capacity and the idleness negatively affect the general exhibitions. The data transfer capacity parleys what number of streams the Controller can process, just as the misfortune rate if the framework is under substantial burden while the dormancy drives the general conduct of the system. Regardless of whether the proposed arrangements can be executed effectively, an upstream assessment of the utilization of the system is vital. A perfect circumstance would permit a high throughput and if the inertness is sufficiently low, the cushion attributes are not significant. In any case, keeping a raised throughput in a high inactivity circumstance causes heartbreaking exhibitions; one of the arrangements is to constrain the throughput by various methods which in turn lessen the general execution of the system. The exhibition examination is finished by looking at all system topologies on premise of data transfer capacity usage, bundle transmission rate, full circle spread postponement between end hubs and most extreme got throughput. The structure of every OpenFlow-empowered configuration is finished utilizing model system emulator called Mininet.Here in order to implement SDNC virtual box VM Ware has been installed in windows in that ubuntu has been installed in that mininet has been installed. The basic commands of mininet are.

$$
\text { sudo mn }
$$

it consists of I controller , 1 switch and two hosts and other commands such as

$$
\text { sudo mn -topo minimal }
$$

is the minimal topology used to check the topology and other four topologies such as Linear, tree, reversed, and single topology has been executed and in that topology the values are shown in the figures respectively. 


\section{Results and Discussions}

Right now, similar execution examination of topologies like single topology, linear topology, tree topology, reversed and minimal topology are taken into consideration after executing the system. This are done by assuming data communication use, parcel transmittance rate, period of time which is needed to transfer bundles from the source to sink hub and in order to obtain high throughput. Now in this work an exhibition investigation of five topologies is accomplished for a topological planned system of 16 hosts. Transmission capacity usage for each topology is classified in Table. 1 and is appeared in Fig. 4.

The OpenFlow-empowered system engineering for fundamental OpenFlow organize topologies is executed utilizing Mininet: A quick prototyping for programming characterized arrange (Robitza, W., Ahmad, A., Kara, P.A. et al. (2017) . Mininet underpins five worked in different topologies like single, minimal, tree, linear as well as reserved(A Azzouni et al,2016). Default one OS available which is specific one with open flow topology with two hosts as well as reference controller and even they also use order line interface (CLI). Present investigation of a system comparative with data transmission use, deferral of the system, generally speaking system throughput and burden on the system is finished.

Table. 1.Topologies

\begin{tabular}{|c|c|c|c|c|c|c|}
\hline SI No & Network Topologies & Single & Linear & Tree & Reversed & Minimal \\
\hline 1 & No: of controllers & 1 & 1 & 1 & 1 & 1 \\
\hline 2 & $\begin{array}{c}\text { No: of Open flow Enabled } \\
\text { Switches }\end{array}$ & 1 & 16 & 5 & 7 & 6 \\
\hline 3 & Number of Hosts & 16 & 16 & 16 & 16 & 16 \\
\hline 4 & $\begin{array}{c}\text { Maximum Utilized } \\
\text { Bandwidth(sec) }\end{array}$ & 4.2 & 3.85 & 4.75 & 4.6 & 4.8 \\
\hline 5 & $\begin{array}{c}\text { Minimum Utilized } \\
\text { Bandwidth(sec) }\end{array}$ & 3.8 & 2.9 & 4 & 4.5 & 4.6 \\
\hline
\end{tabular}

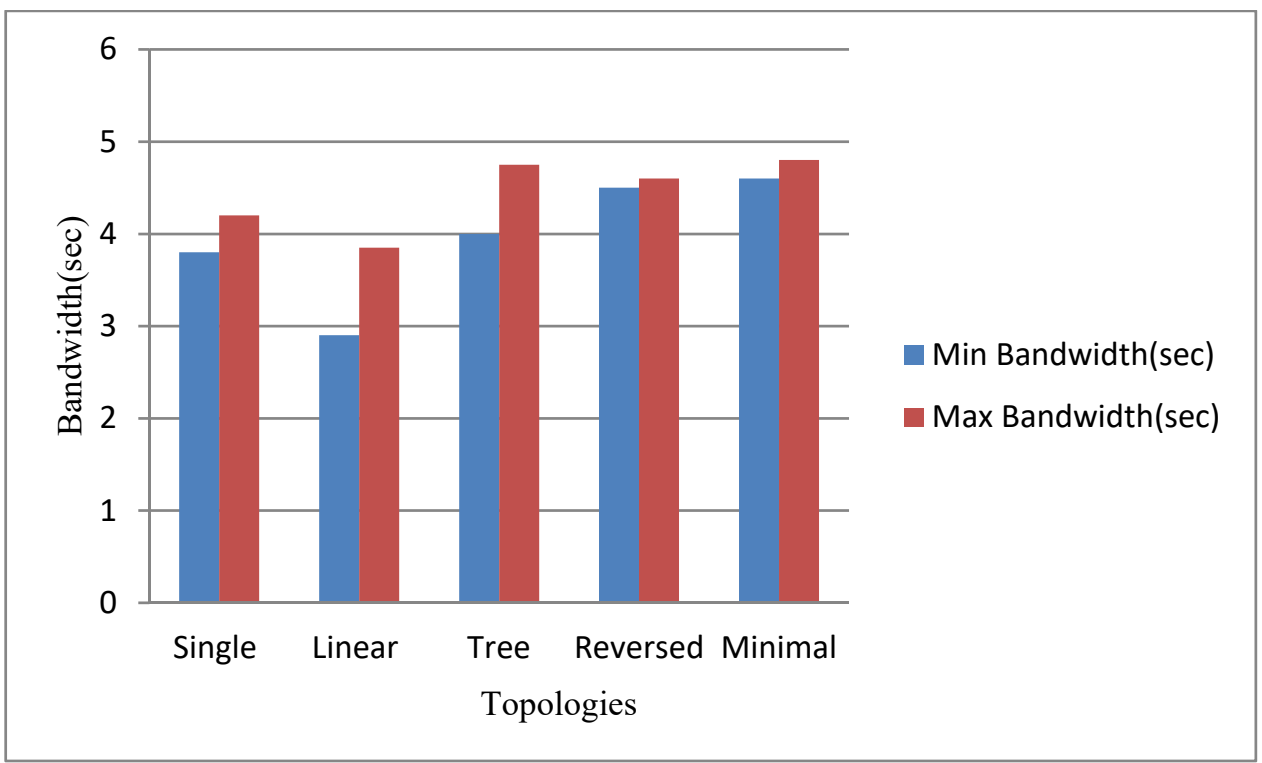

Fig. 4. Bandwidth utilization for basic Open Flow topologies

A near presentation examination of Single, Linear, Tree, Reversed and Minimal Topologies, are done dependent on outcome after execution of systems. The exhibition examination is finished by looking at all system topologies here, an exhibition investigation for the three topologies as talked about in past segment is accomplished for 16 hosts. Data transfer capacity use for every topology is arranged in Table. 1 and is appeared in Fig. 4. The recreation results acquired for data transfer capacity usage of the system is by executing 'iperf' (Rowshanrad, S., Abdi, V., $\&$ Keshtgari, $M$,2016) direction in Mininet. The quantity of this switches required for usage of single, straight, tree, turned around and minimal topology systems for a typical 16 hosts design is 1, 6, 16, 4.8 and 4.6 individually. In Fig. 5, four topologies compared and in that linear having less bandwidth. The bandwidth used in linear topology is minimum as well as tree topology is maximum based on the solution obtained.One host is linked to 
one switch and the interconnection are connected to each other for the interconnectivity to terminal nodes. For the number of nodes is limited to the total bandwidth of use. The architecture is spread in tree topology but regulated as shown centrally in Fig. 5.The average bandwidth consumption is more.

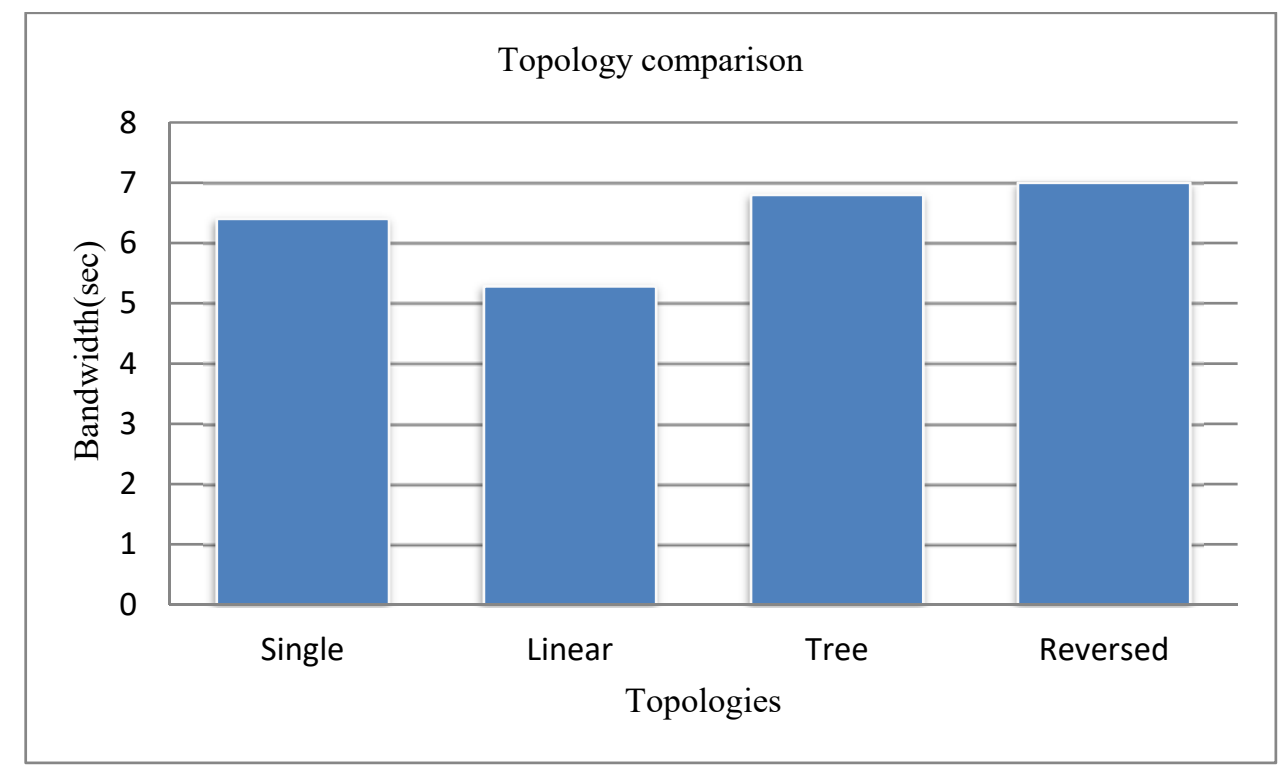

Fig. 5. Comparison of four topologies

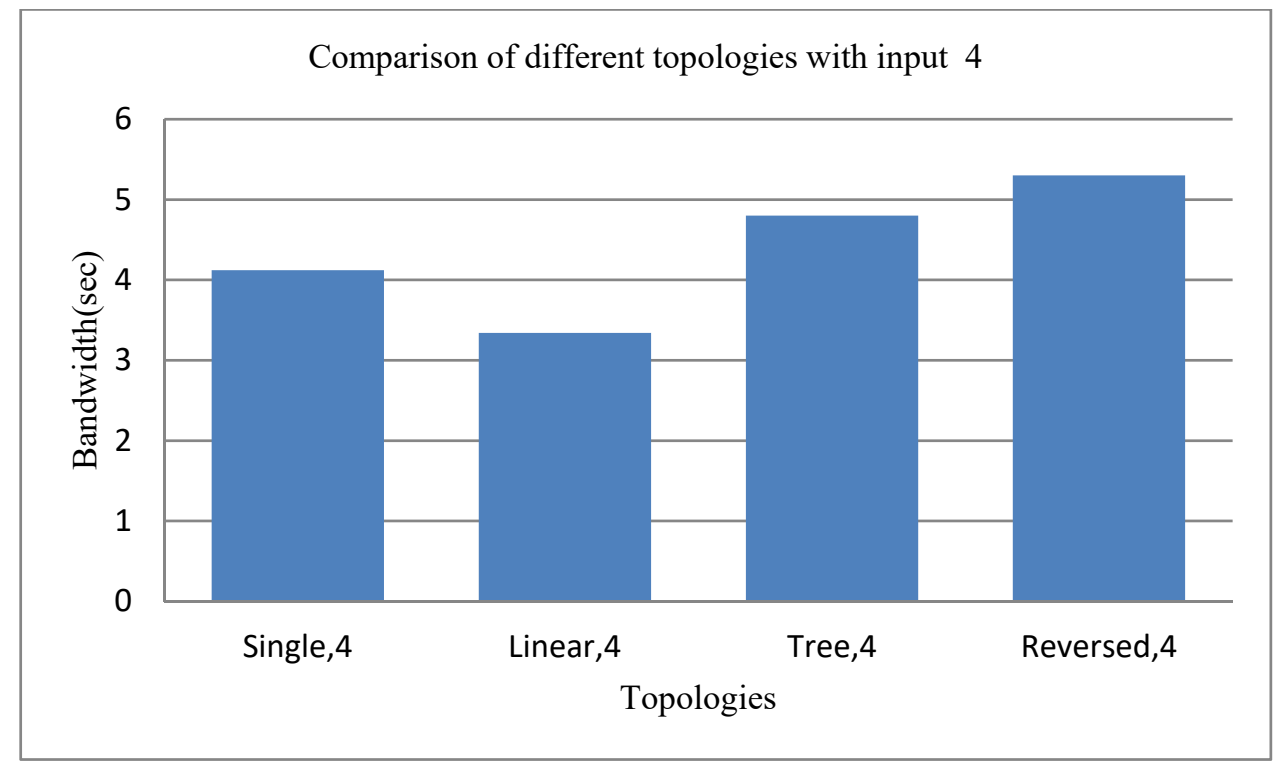

Fig. 6. Comparison of four topologies with input 4

In Fig. 6, sudo mn -topo linear,4 ,sudo mn -topo reveresed,4,sudo mn -topo single,4, sudo mn -topo tree, 4 has been compared in that linear having low bandwidth. Similarly sudo $\mathrm{mn}$-topo linear, 8 ,sudo $\mathrm{mn}$-topo reveresed, 8 , sudo $\mathrm{mn}$-topo single, 8 , sudo $\mathrm{mn}$-topo tree, 8 has been compared in that also linear having less bandwidth as shown in Fig. 7. 
Table. 2. Open flow Protocol Comparison

\begin{tabular}{|c|c|c|c|c|c|}
\hline SI No & Network Controllers & POX & NOX & FloodLight & Openflow \\
\hline 1 & No: of controllers & 1 & 1 & 1 & 1 \\
\hline 2 & $\begin{array}{c}\text { No: of Open flow Enabled } \\
\text { Switches }\end{array}$ & 1 & 1 & 1 & 1 \\
\hline 3 & Number of Hosts & 2 & 2 & 2 & 2 \\
\hline 4 & $\begin{array}{c}\text { Maximum Utilized } \\
\text { Bandwidth(sec) }\end{array}$ & 0.6 & 0.5 & 0.7 & 0.07 \\
\hline
\end{tabular}

From these qualities straight topology can be taken as contribution for open flow convention and the open stream has been contrasted and various conventions, for example, floodlight, pox, nox and appeared in Table. 2 and comprehend that open flow having inactivity low. After these bundles has been checked in parcel sifting instruments and found that bundle misfortune is less as appeared in Table. 3. Fig. 8 shows the throughput where term in sec wherein bundles misfortune is less. Fig. 9 and Fig. 10 are yield for throughput from the parcel filtering and broke down that the bandwidth, latency and throughput can be improved by utilizing openflow convention in SDNC.

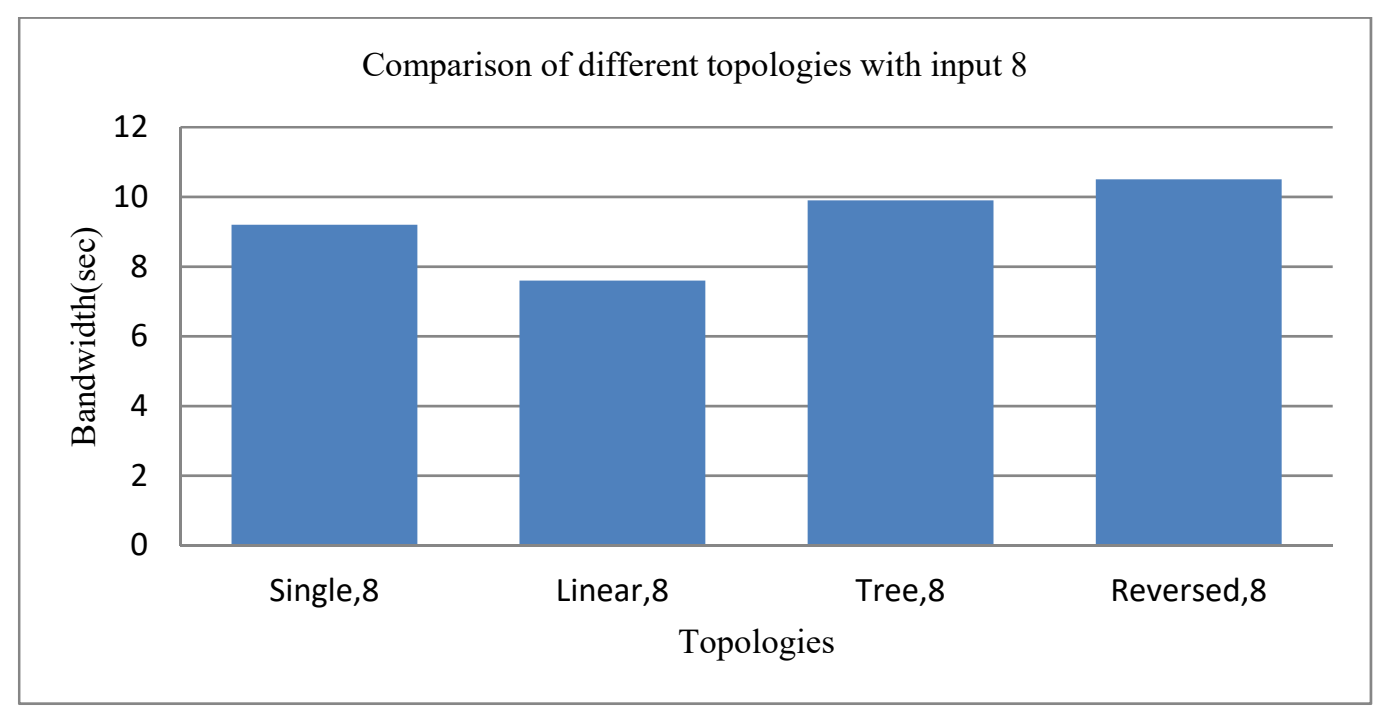

Fig. 7. Comparison of four topologies with input 8

Next, five topology tests are carried out based on the transmission rates of the packet (PTR) for all 5 topologies which is illustrated in Fig. 8 and is discussed in Table. 3,From the result obtained the all-out time taken by the three systems is almost like one another for different parcel transmission numbers. As the performance of a large number of hubs is the same in any OpenFlow arrangement, the OpenFlow organization is dynamic to implement different system topologies for normal bundle transmission.

Table. 3. Output comparison of packet filtering

\begin{tabular}{|c|c|c|c|c|}
\hline Time & 4 week & 3 week & 2 week & 1 week \\
\hline No:of Packets Transferred & 28000 & 21000 & 14000 & 7000 \\
\hline No:of Packets Received & 28990 & 20995 & 13997 & 6998 \\
\hline No:of Packets Lost & 10 & 5 & 3 & 2 \\
\hline Duration(sec) & 0.98 & 0.66 & 0.44 & 0.22 \\
\hline
\end{tabular}




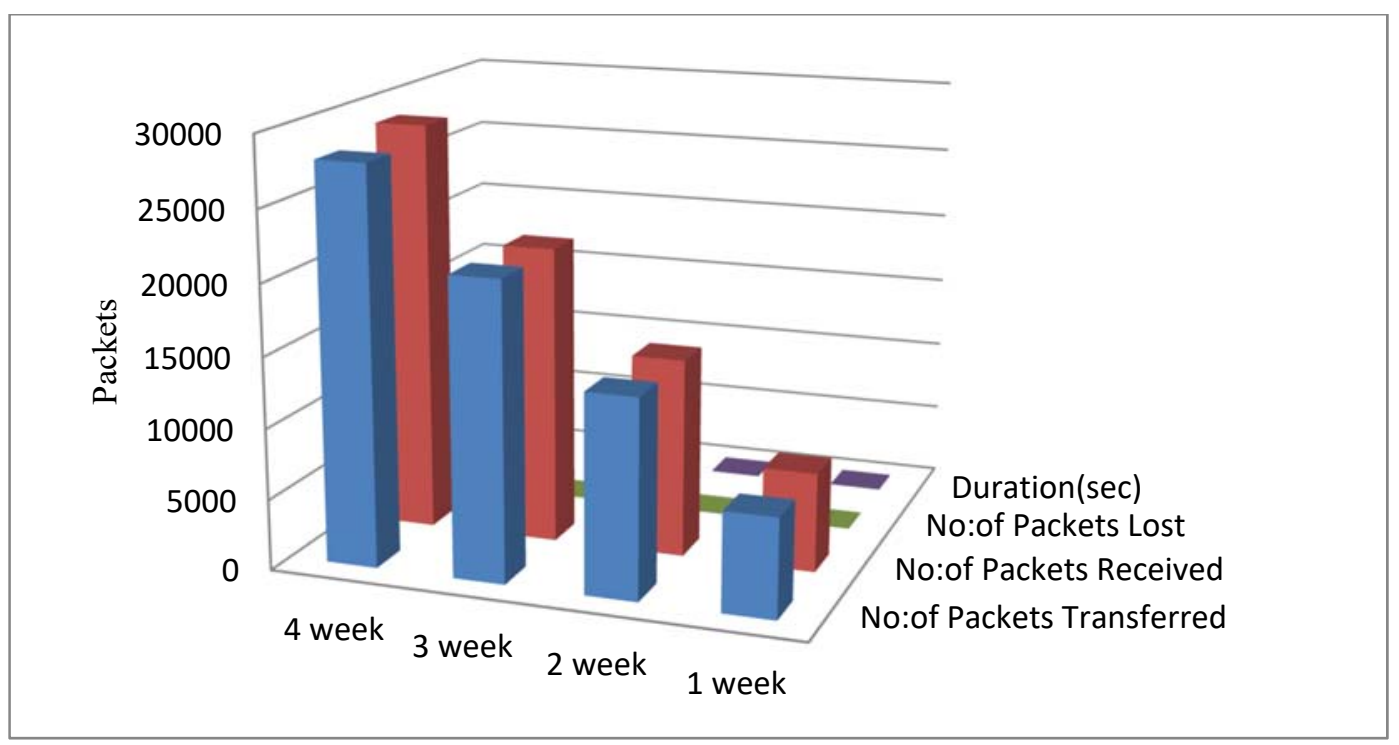

Fig. 8. Throughput

Finally, correlations between this essential topologies are done dependent on Through-put investigation of a system as appeared in Fig. 8 and which is featured as the criterion of data transmitted from source to sink in a specific period of given time which is calculated in bits per second (bps). Logically this is a proportion of greatest beneficiary data transfer capacity to full circle time between hubs: "Throughput $=$ most extreme recipient transmission capacity/full circle time" The general system throughput can be acquired by thinking about transfer speed usage and full circle time as examined is determined and demonstrated graphically in beneath Figures. "Max throughput $=\max$ transfer speed $/ \mathrm{min} \mathrm{rtt} "$ and "Min throughput $=\min$ transmission capacity $/ \mathrm{max} \mathrm{rtt} "$. From the got chart, it is said that the throughput of the straight topology is less when contrasted with different topologies, this is been related on the grounds that the data transfer capacity use is less and the general full circle spread deferral between hubs is additionally more. Though, single topology arrange is having most extreme throughput when contrasted with different topologies, this is on the grounds that the single have only one switch and between has 2 bounces. With these the single also delays the process and the amount of through-put is less. Throughput from the parcel separating is appeared in . The yield from the bundle sifting is appeared in Fig. 10.

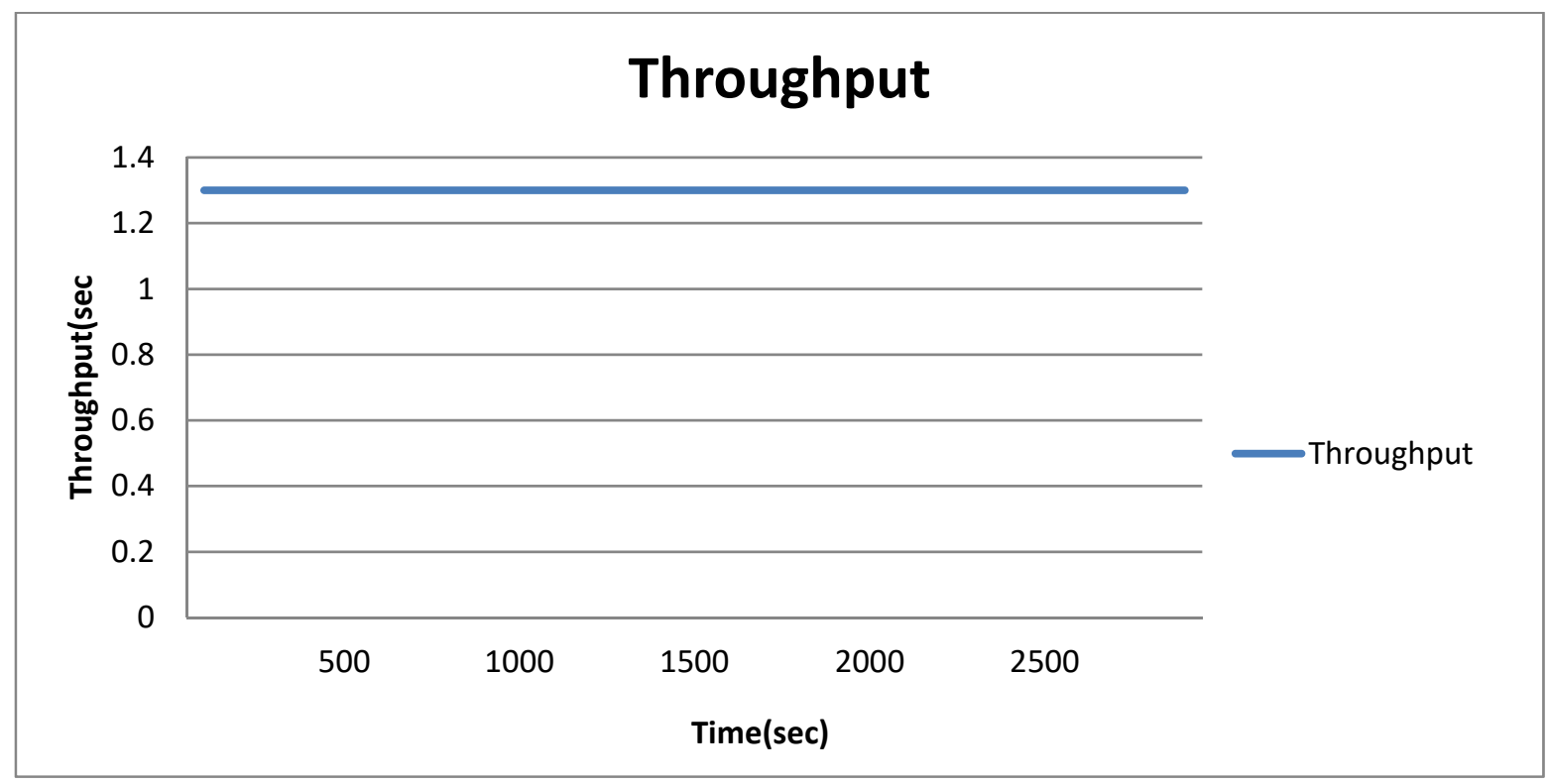

Fig. 9. Throughput from the packet filtering 


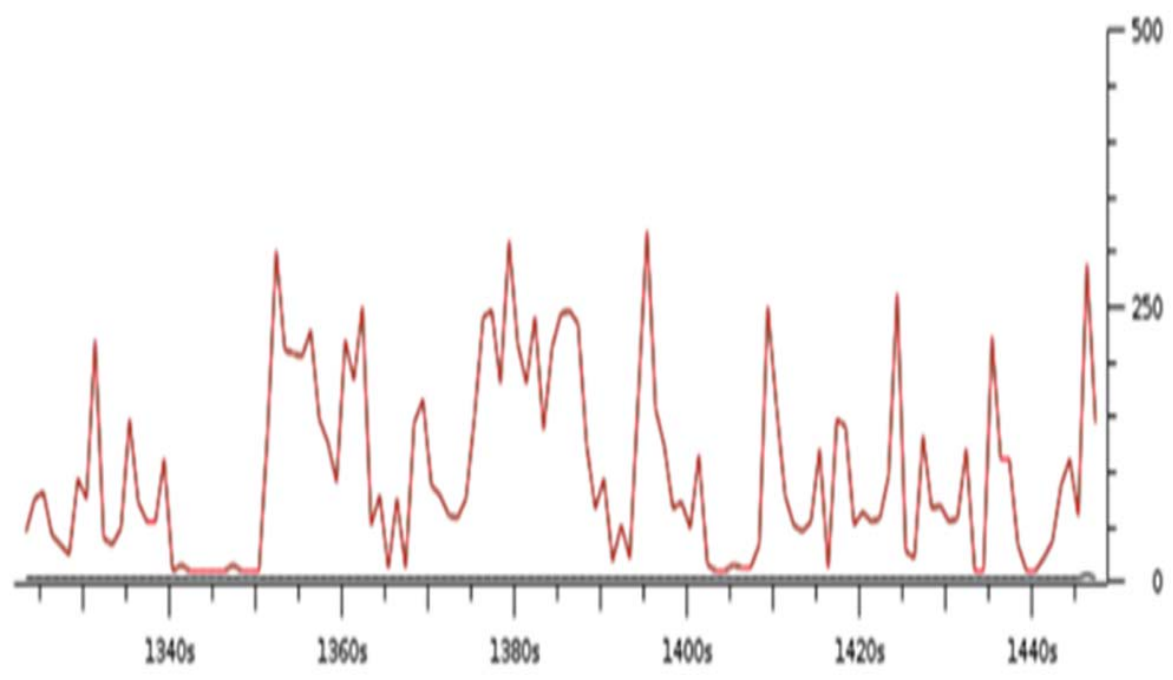

Fig. 10. Output from the packet filtering

\section{Conclusion}

Based on execution done on the investigation of the proposed organized topologies, the proposal of the linear one which is better, when contrasted with other 3 tree,reversed and single type, with certain restrictions. On the off chance that in linear topology the quantity of legion in the system will expand then the presentation of the system will consequently get diminish. Whole heap of a system will took care of by a solitary OpenFlow switch, and the stream sections in a switch will be more. In the event that, OpenFlow switch refuses assistance because of some breakdown, than entire system will get obliterated. Likewise, rate of the streams, transmission capacity, bundle falling likelihood, and a lot increased physical parameters which will get influenced because of the restricted equipment requirements. As it was appeared, a wide range of explores are attempting to give quicker and progressively dependent base for $5 \mathrm{G}$ remote systems. SDN as the principle segment of giving the virtualization picked up progressively fascination. The handover postponement ought to in a perfect world be 0.7 seconds, when both arranging and execution stages are incorporated. And no more, a deferral of as long as two minutes during handover among 3G and LTE systems can be suited. By utilizing SDN design, the information way should be characterized over OpenFlow convention and the preparing time for any OpenFlow message diminishes essentially, which brings about decreasing the idleness at the handover impressively by roughly 30 percent and takes into consideration a quicker handover. Furnishing progressively adaptable system with high rate throughput is as yet should have been more researched.

\section{References}

[1] Agyapong,P K;Iwamura,M;Staehle,D;Kiess,W;Benjebbour,A.(2014), "Design considerations for a 5G network architecture," in IEEE Communications Magazine, vol. 52, no. 11, pp. 65-75.

[2] Akshay,J;Lopez-Aguilera,E;Demirkol,E.(2017), "Mobility management as a service for 5G networks", IEEE ISWCS 2017 Work, pp. $1-6$.

[3] Azzouni,A;et al.(2016), "Fingerprinting OpenFlow controllers: The first step to attack an SDN control plane", GLOBECOM.

[4] Balasubramanian,V; Zaman,F; Aloqaily,M; Ridhawi,Y; Jararweh,H. B;Salameh.(2019), "A Mobility Management Architecture for Seamless Delivery of 5G-IoT Services," ICC 2019 - 2019 IEEE International Conference on Communications (ICC), Shanghai, China, pp. 1-7.

[5] Cox,J H;Clark,R J;Owen,H L(2016), "Leveraging SDN to improve the security of DHCP," in Proceedings of the 2016 ACM International Workshop on Security in Sofware Defned Networks and Network Function Virtualization (SDNNFV-SEC 2016), pp. 3538.

[6] Dotcenko,S;Vladyko,S;Letenko,I.(2014), "A fuzzy logic-based information security management for software-defined networks," 16th International Conference on Advanced Communication Technology, Pyeongchang, pp. 167-171

[7] Ernardos,C.J;De La Oliva;Serrano,A;Anchs,P;Contreras,A;Hao Jin niga,L M.(2014), "An architecture for software defined wireless networking," Wireless Communications, IEEE, vol.21, no.3, pp.52,61.

[8] ETSI MCC Department(2014), Standardization of Machine-type Communications V0.2.4 (2014-06).

[9] ETSI MCC Department(2013),3GPP Multimedia Broadcast and Multicast Service (MBMS) V0.0.2 (2013-04)

[10] European Commission(2013), "Advanced 5G Network Infrastructure for the Future Internet - Public Provate Partnership in Horizon 2020,” H2020 LEIT, Work Programme, ICT 14.

[11] 3GPP TS 23.401, "General Packet Radio Service (GPRS) enhancements for Evolved Universal Terrestrial Radio Access Network (EUTRAN) access."

[12] Guerzoni,R;Trivisonno,R;Vaishnavi,I;Despotovic,Z;Hecker,A;Beker,S;Soldani,D.(2014), "A novel approach to virtual networks embedding for SDN management and orchestration," IEEE Network Operations and Management Symposium (NOMS), Krakow, pp. $1-7$.

[13] Hucheng,W;Chen,S;Ming,S C A;Yan,S.(2017),'Mobility driven network slicing: an enabler of on demand mobility management for 5G”, J. China Univers. Posts Telecommun., 24 (4) , pp. 16-26

[14] Huiqiang Yuwen, Liancheng Zhang, Zhenxing Wang and Yazhou Kong(2016), "Probability-based delay scheme for resisting SDN scanning," 2nd IEEE International Conference on Computer and Communications (ICCC), Chengdu, pp. 1096-1101. 
[15] METIS(2013), "Scenario, Requirements and KPIs for 5G Mobile and Wirelss System (Deliverable D.1.1)"

[16] Mohammadi,R;Javidan,R;Conti,M.(2017), "SLICOTS: An SDN-Based Lightweight Countermeasure for TCP SYN Flooding Attacks," in IEEE Transactions on Network and Service Management, vol. 14, no. 2, pp. 487-497.

[17] Mueller,Y;Chen,B;Reichel,V;Vlad,Magedanz,T.(2014),"Design and implementation of a Carrier Grade Software Defined Telecommunication Switch and Controller," IEEE Network Operations and Management Symposium (NOMS), Krakow, pp. 1-7.

[18] " Link-Level Access Cloud Architecture Design Based on SDN for 5G Networks"IEEE Network,pp- 0890-8044

[19] Rietz, A; Brinner,Cwalinsky,R.(2015), "Improving Network Security in Virtualized Environments with Openflow"

[20] Rowshanrad,S;Abdi,V;Keshtgari,M.(2016).Performance Evaluation Of SDN Controllers: Floodlight and Opendaylight. IIUM Engineering Journal, 17(2),pp-47-57

[21] Robitza,W;Ahmad,A; Kara, P.A; et al. (2017)Challenges of future multimedia QoE monitoring for internet service providers. Multimed Tools Appl 76, pp-22243-22266.

[22] Shirali-Shahreza,S;Ganjali,Y.(2018),"Protecting Home User Devices with an SDN-Based Firewall," in IEEE Transactions on Consumer

[23] Wang,j;Chen,Y.(2017), "An SDN-based defensive solution against DHCP attacks in the virtualization environment," IEEE Conference on Dependable and Secure Computing, Taipei, 2017, pp. 529-530.

[24] Yazıc1,V;Kozat,U C;Sunay,M O.(2014), "A new control plane for 5G network architecture with a case study on unified handoff, mobility, and routing management," in IEEE Communications Magazine, vol. 52, no. 11, pp. 76-85

[25] Zhao,Z;Liu,F;Gong,D;Chen,L;Xiang,F;Li,Y.(2017), "An SDN-based IP hopping communication scheme against scanning attack," 2017 IEEE 9th International Conference on Communication Software and Networks (ICCSN), Guangzhou, pp. 559-564. 